\title{
MUTATION AND POPULATION SIZE
}

\author{
BRYAN CLARKE \\ Genetics Department, University Park, Nottingham NG7 2RD
}

Received 18.xii.72

\begin{abstract}
SUMMARY
A combination of ecological and genetic models makes it possible to predict the effects of mutation on population size. Although all disadvantageous mutants produce the same genetic load (as conventionally defined) different types of mutants may have different effects upon the numerical equilibrium. Those reducing competitive ability can cause an increase in population size, despite the fact that they are eliminated by natural selection. When the selective values of genotypes depend upon their frequencies (or densities), conventional genetic load measures neither the effect of mutation on the rate of increase of the population nor its effect on the total numbers at equilibrium. If the consequences of mutation are to be adequately understood, the ecological parameters must be taken into consideration.
\end{abstract}

\section{INTRODUCTION}

SINCE the work of Haldane (1937) and Muller (1950), much has been written about the "genetic load" caused by mutation. This mutational load has been defined as the fraction by which the mean fitness of a population subject to mutation differs from the fitness of the wild, non-mutant, genotype (Crow, 1970). The operational definition of load is then:

$$
L=\frac{W_{\max }-\bar{W}}{W_{\max }}
$$

where $W_{\max }$ is the fitness (selective value) of the non-mutant genotype, and $\bar{W}$ the mean fitness (or average selective value) of the population subject to mutation.

In discussions of mutational load it has often been supposed, implicitly or explicitly, that the average selective value is necessarily an indicator of the relative fitness or competitive ability of a population compared to other populations. This supposition is incorrect when selective values are defined, as they usually are, only in relative terms. Relative values within populations cannot be extrapolated to comparisons between populations (see Cain and Sheppard, 1954; $\mathrm{Li}, 1955)$. Another pitfall has been the assumption that the genetic load is a measure of factors directly related to population size (see Turner and Williamson, 1968; Wallace, 1970).

The present paper describes a study of the effects of mutation on population size. The study has developed from earlier work on density-dependent selection (Williamson, 1957; Barber, 1965; Roughgarden, 1971; Charlesworth, 1971; King and Anderson, 1971; Clarke, 1972).

\section{Models of NatUral SELEGTion}

The conventional model of natural selection assumes a large randommating population with discrete generations and without immigration or 
emigration. Let us suppose that within this population there are two alleles $M$ and $m$ at an autosomal locus, and that $M$ is completely dominant to $m$. The frequency of $M$ among the reproducing adults of one generation is $p$, and the frequency of $m$ is $q(p+q=1)$.

We assume that $M$ is the "wild-type" gene and that $m$ is the mutant. The mutation rate from $M$ to $m$ is $\mu$. When $m$ is rare we can ignore backmutation from $m$ to $M$. If mutation occurs during the formation of gametes and if all three genotypes have equal fertility, the gene frequency of $m$ among the gametes will be:

$$
q^{\prime}=q+\mu(1-q)
$$

With random mating the frequencies of zygotes will be

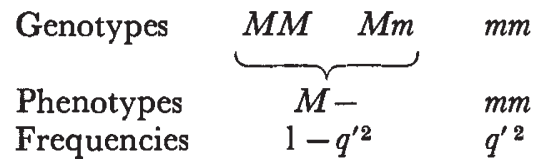

When the phenotypes have different probabilities of surviving to produce offspring, represented by the selective values $a$ (for $M-$ ) and $c$ (for $\mathrm{mm}$ ), the phenotype frequencies after selection will be

$\begin{array}{lll}\text { Phenotypes } & M- & m m \\ \text { Frequencies after selection } & a\left(1-q^{2}\right) & c q^{\prime 2}\end{array}$

The gene frequency after selection is then

$$
q^{\prime \prime}=\frac{q^{\prime 2}(c-a)+a q}{q^{\prime 2}(c-a)+a}
$$

and the overall change of gene frequency

$$
\Delta q=q^{\prime \prime}-q=\frac{\left(1-q^{\prime}\right)\left[q^{\prime 2}(c-a)+a \mu\right]}{(1-\mu)\left[q^{\prime 2}(c-a)+a\right]}
$$

When there is a balance between mutation and selection $\Delta q$ is zero and thus

$$
q^{2}(c-a)+a \mu=0
$$

from which

$$
\hat{q}^{\prime}=\sqrt{\frac{a \mu}{a-c}}
$$

where $q^{\prime}$ is the non-trivial equilibrium value of $q^{\prime}$. This equation is a generalisation of the one used by Muller (1950).

Noting that the average selective value $(W)$ is equal to

$$
q^{\prime 2}(c-a)+a,
$$

we can calculate the genetic load at equilibrium from equation (1)

$$
L=\frac{a-\left[\hat{q}^{\prime 2}(c-a)+a\right]}{a}=\mu .
$$

The selective values ( $a$ and $c$ ) are conventionally defined as measures only of relative fitness. The equations given above, however, are equally 
valid if $a$ and $c$ are absolute fitnesses. In this event they measure changes in the numbers of each genotype from generation to generation. The average selective value $(W)$ is then related to the overall change in numbers $(\Delta \mathcal{N})$ as follows:

$$
\Delta \mathcal{N}=\mathcal{N}(\bar{W}-1)
$$

and in the present situation

$$
\Delta \mathcal{N}=\mathcal{N}\left[q^{\prime 2}(c-a)+a-1\right] .
$$

If the population is numerically at equilibrium, $\Delta \mathcal{N}=0$ and therefore

$$
q^{2}(c-a)+a-1=0 .
$$

When the population is at equilibrium for both numbers and gene frequency, we can treat (2) and (5) as simultaneous equations, from which

$$
\begin{aligned}
& a=\frac{1}{1-\mu} \\
& c=\frac{q^{\prime 2}-\mu}{q^{\prime 2}(1-\mu)} .
\end{aligned}
$$

The equations derived in this section are general, and can be applied whether the selective values are constant or variable. If the selective values are functions of density, equations (6) and (7) can be used to derive the value of $\mathcal{N}$ at equilibrium. Thus we can solve $\hat{\mathcal{N}}$ as well as $q^{\prime}$. The precise result will depend upon the form of the relation between selective value and density. This is discussed below.

\section{MODELS OF POPULATION GROWTH}

The logistic model of population growth and competition due to Verhulst (1844), and elaborated by Lotka (1932) and Volterra (1926), has been used extensively. In one form it can be expressed as

$$
\frac{d \mathcal{N}}{d t}=r \mathcal{N}\left(\frac{K-\mathcal{N}}{\kappa}\right)
$$

Where $\mathcal{N}$ is the population size, $r$ is a constant representing the intrinsic rate of natural increase, and $K$ represents the maximum population size permitted by the environment (often called the "carrying capacity"). The density-dependent component of growth is represented by the factor

$$
\frac{\kappa-\mathcal{N}}{\kappa}
$$

When two species are competing the model takes the form of simultaneous equations (Volterra, 1926; Lotka, 1932):

$$
\begin{aligned}
& \frac{d \mathcal{N}_{1}}{d t}=r_{1} \mathcal{N}_{1}\left(\frac{\kappa_{1}-\mathcal{N}_{1}-\alpha_{1} \mathcal{N}_{2}}{\kappa_{1}}\right) \\
& \frac{d \mathcal{N}_{2}}{d t}=r_{2} \mathcal{N}_{2}\left(\frac{\kappa_{2}-\mathcal{N}_{2}-\alpha_{2} \mathcal{N}_{1}}{\kappa_{2}}\right)
\end{aligned}
$$

where the subscripts refer to individual species and $\alpha_{1}$ and $\alpha_{2}$ are the 
competition coefficients representing the inhibitory effects of each species on the other.

Although equation (8) sometimes adequately represents the sigmoid growth curve of populations there are others that may serve equally well (see Williamson, 1972). The following equation, chosen for reasons that will become apparent, is one example among many:

$$
\frac{d \mathcal{N}}{d t}=\mathcal{N}\left(\frac{w k}{k+w \mathcal{N}}-1\right)
$$

where $w$ and $k$ are constants whose effects are similar to those of $r$ and $K$. Using this model, competition between two species can be represented as

$$
\begin{aligned}
& \frac{d \mathcal{N}_{1}}{d t}=\mathcal{N}_{1}\left(\frac{w_{1} k_{1}}{k_{1}+w_{1} \mathcal{N}_{1}+\alpha_{1} w_{2} \mathcal{N}_{2}}-1\right) \\
& \frac{d \mathcal{N}_{2}}{d t}=\mathcal{N}_{2}\left(\frac{w_{2} k_{2}}{k_{2}+w_{2} \mathcal{N}_{2}+\alpha_{2} w_{1} \mathcal{N}_{1}}-1\right) .
\end{aligned}
$$

\section{Combining the genetra AND egologtaAl models}

If we suppose that population growth is adequately represented by the Verhulst equation (8), we can relate it to the average selective value by constructing a difference equation rather than a differential (i.e. by assuming discrete generations).

$$
\Delta \mathcal{N}=R_{\max } \mathcal{N}\left(1-\frac{\mathcal{N}}{K}\right)
$$

where $R_{\max }$ is the maximum net reproductive rate (or intrinsic rate of increase per generation).

Consequently, from equation (4)

and

$$
\mathcal{N}(\bar{W}-1)=R_{\max } \mathcal{N}\left(1-\frac{\mathcal{N}}{K}\right)
$$

$$
\bar{W}=1+R_{\max }\left(1-\frac{\mathcal{N}}{K}\right)
$$

Unfortunately, this equation is not satisfactory because the right-hand side may become negative when $\mathcal{N}$ is large, and the genetical model does not allow negative selective values.

We can escape from the problem by using equation (11), from which

$$
\bar{W}=\frac{w k}{k+w \mathcal{N}}
$$

Here the right-hand side is always positive, and varies between zero (when $\mathcal{N}=\infty$ ) and $w$ (when $\mathcal{N}=0$ ). Note that this equation resembles the Michaelis-Menten equation in enzyme kinetics, and will be designated the Michaelis model.

An alternative escape, used by Roughgarden (1971) and Charlesworth (1971), is to truncate equation (14) so that when

it is assumed to be zero.

$$
1+R_{\max }\left(1-\frac{\mathcal{N}}{K}\right)<0
$$


In the following investigation I will concentrate upon the Michaelis model based on equation (15), but will also discuss the consequences of the logistic model based on equation (14).

\section{The joint (Miahaelis) MOdel}

I assume that $\mathcal{N}$ represents the number of fertile adults, that the fertility and fecundity of all genotypes are equal, and that young are produced in an excess (represented by the constant $E$ ). They are then subject to densityindependent mortality represented by the selective values $v_{1}$ (for $M-$ ) and $v_{2}$ (for $\mathrm{mm}$ ). The combined effects of $E$ and $v_{1}$, or $E$ and $v_{2}$, are represented by the " intrinsic selective values" $w_{1}$ and $w_{2}\left(w_{1}=E v_{1}>1 ; w_{2}=E v_{2}>1\right)$.

Density-dependent selective mortality is assumed to occur after densityindependent selection, but the consequences of reversing this order are considered below. For the purposes of defining density-dependent selective values, the two phenotypes $(M-$ and $m m$ ) are treated as if they were competing species. The selective values are then derived from equations (12) and (13) in a manner corresponding to the derivation of equation (15).

The density-dependent selective values are then

Phenotype

$M-$

$m m$

Density-dependent selective value

$$
\frac{k_{1}}{k_{1}+w_{1} \mathcal{N}\left(1-q^{2}\right)+\alpha_{1} w_{2} \mathcal{N} q^{2}} \frac{k_{2}}{k_{2}+w_{2} \mathcal{N} q^{2}+\alpha_{2} w_{1} \mathcal{N}\left(1-q^{2}\right)} .
$$

Combining both types of selection, we obtain the overall selective values ( $a$ and $c$ ) which represent proportional increases or decreases of numbers for each phenotype.

Phenotype $M-\quad m m$

Overall selective value

$$
a=\frac{w_{1} k_{1}}{k_{1}+w_{1} \mathcal{N}\left(1-q^{2}\right)+\alpha_{1} w_{2} \mathcal{N} q^{2}} \quad c=\frac{w_{2} k_{2}}{k_{2}+w_{2} \mathcal{N} q^{2}+\alpha_{2} w_{1} \mathcal{N}\left(1-q^{2}\right)} .
$$

These selective values can then be applied to the calculation of $\hat{\mathcal{N}}$ and $q$, using equations (6) and (7).

A consideration of the overall selective values (16) shows that a mutant could be disadvantageous in three different ways. It could have a reduced intrinsic selective value $(w)$, a reduced carrying capacity $(k)$, or a reduced competitive ability (an increased value of $\alpha_{2}$ or a decreased value of $\alpha_{1}$ ). Natural selection against mutants with reduced $w$ is equivalent to the $r$ selection of MacArthur (1962), and selection against mutants with reduced $k$ is equivalent to his $K$-selection. Selection against mutants with a changed value of $\alpha$ has not been defined before, although it may correspond to the "soft" selection of Wallace (1970). I shall call it $\alpha$-selection.

Because the three kinds of selection may have different effects on population numbers, the distinction between them is important.

\section{THE NUMERICAL LOAD}

The proportional reduction in numbers (the numerical load) brought 
about by mutation to a deleterious allele can be defined in a way analogous to the definition of the conventional genetic load:

$$
\mathcal{N} L=\frac{\hat{\mathcal{N}}_{0}-\hat{\mathcal{N}}_{\text {mut }}}{\hat{\mathcal{N}}_{0}}
$$

where $\hat{\mathcal{N}}_{0}$ is the numerical equilibrium in the absence of mutation, and $\hat{\mathcal{N}}_{\text {mut }}$ is the equilibrium in its presence.

(a) The numerical load for mutants with a reduced intrinsic selective value

If we suppose that the mutant differs from the wild type only in the value of $w$, we can write

$$
\begin{aligned}
w_{1} & =w \\
w_{2} & =w(1-s) \\
k_{1} & =k_{2}=k \\
\alpha_{1} & =\alpha_{2}=1 .
\end{aligned}
$$

(Note that if $\alpha_{1}$ and $\alpha_{2}$ are less than 1 the system may become one of balanced polymorphism. The exact conditions are given by Clarke, 1972.)

Thus

$$
a=\frac{w k}{k+w \mathcal{N}\left(1-s q^{\prime 2}\right)} \quad c=\frac{w k(1-s)}{k+w \mathcal{N}\left(1-s q^{\prime 2}\right)}
$$

and from (6) and (7) at equilibrium,

and

$$
\frac{1}{1-\mu}=\frac{w k}{k+w \mathcal{N}\left(1-s \hat{q}^{\prime 2}\right)}
$$

$$
\frac{{q^{\prime 2}}^{2}-\mu}{q^{\prime 2}(1-\mu)}=\frac{w k(1-s)}{k+w \hat{\mathcal{N}}\left(1-s q^{\prime 2}\right)}
$$

from which we obtain

and

$$
q^{\prime}=\sqrt{\frac{\mu}{s}}
$$

$$
\hat{\mathcal{N}}_{\text {mut }}=\frac{k[w(1-\mu)-1]}{w(1-\mu)}
$$

Now $\hat{\mathcal{N}}_{0}$ is obtained by setting $\hat{q}^{\prime}=0$ and $a=1$, from which we obtain

$$
\hat{\mathcal{N}}_{0}=\frac{k(w-1)}{w} \text {. }
$$

Therefore the numerical load is

$$
\mathcal{N} L=\frac{\mu}{(w-1)(1-\mu)} .
$$

(b) The numerical load for mutants with a reduced carrying capacity

Here we write $k_{1}=k, k_{2}=k\left(1-s^{\prime}\right), w_{1}=w_{2}=w, \alpha_{1}=\alpha_{2}=1$, and obtain, by a procedure similar to the last;

$$
\hat{q}^{\prime}=\sqrt{\frac{\mu\left[w(1-\mu)-s^{\prime}\right]}{s^{\prime}[w(1-\mu)-1]}}
$$


and

$$
\hat{\mathcal{N}}_{\text {mut }}=\frac{k[w(1-\mu)-1]}{w}
$$

so that the numerical load is

$$
\mathcal{N} L=\frac{w \mu}{w-1}
$$

\section{(c) The numerical load for mutants with a reduced competitive ability}

If $w_{1}=w_{2}=w$ and $k_{1}=k_{2}=k$, the relative selective values of the genotypes can still be changed by altering $\alpha_{1}$ and $\alpha_{2}$. If both $\alpha_{1}$ and $\alpha_{2}$ are less than unity the system will produce a balanced polymorphism. If, however $\alpha_{1}=1, \alpha_{2}>1$ or $\alpha_{1}<1, \alpha_{2}=1$ the mutant will always be disadvantageous.

Supposing $\alpha_{1}=1$ and $\alpha_{2}=1+s^{\prime \prime}$, we find that $\hat{q}^{\prime}$ is obtained by solving

and that

$$
s^{\prime \prime}\left(1-\hat{q}^{\prime 2}\right)\left(\hat{q}^{\prime 2}-\mu\right)[w(1-\mu)-1]-\mu w(1-\mu)=0
$$

$$
\hat{\mathcal{N}}_{\text {mut }}=\frac{k[w(1-\mu)-1]}{w} .
$$

Noting that this is identical to (18), it follows that the numerical load is identical to (19), i.e.

$$
\mathcal{N} L=\frac{w \mu}{w-1}
$$

If, on the other hand $\alpha_{1}=1-s^{*}$ and $\alpha_{2}=1$, we find that $q^{\prime}$ is obtained by solving

$$
s^{*} q^{\prime 4}[w(1-\mu)-1]+\mu s^{*} \dot{q}^{\prime 2}-\mu w(1-\mu)=0
$$

and that approximately

therefore

$$
\hat{\mathcal{N}}_{\text {mut }}=\frac{k(w-1)[w(1-\mu)-1]}{w\left[w-1-\sqrt{s^{*} \mu w(w-1)}\right]}
$$

$$
\mathcal{N} L \bumpeq \frac{\mu w-\sqrt{s^{*} \mu w(w-1)}}{w-1-\sqrt{s^{*} \mu w(w-1)}} .
$$

Note that this is negative if $s^{*}>\mu$ (i.e. if there is a balance between mutation and selection). I am grateful to Mr L. Nunney for pointing out that the apparent possibility of positive values, when $w$ is small, is the result of the approximation.

\section{The consequences of an alternative model (THE logistic)}

Because the calculation of $\hat{\mathcal{N}}$ depends upon the precise relationship between selection and density, there is no equation for numerical load that is independent of the ecological model chosen to represent population growth. As I have already pointed out, the choice of model is necessarily somewhat arbitrary because we have as yet insufficient knowledge of population ecology. It therefore seems desirable to examine the consequences of an alternative model. 
Roughgarden (1971) and Charlesworth (1971) have represented densitydependent selection by equations similar to (9) and (10). We can apply this logistic model to our present problem and obtain the overall selective values:

$$
\begin{aligned}
& a=w_{1}\left[1+\rho_{1}\left(1-\frac{w_{1}\left(1-q^{2}\right) \mathcal{N}+\alpha_{1} w_{2} q^{2} \mathcal{N}}{K_{1}}\right)\right] \\
& c=w_{2}\left[1+\rho_{2}\left(1-\frac{w_{2} q^{2} \mathcal{N}+\alpha_{2} w_{1}\left(1-q^{2}\right) \mathcal{N}}{K_{2}}\right)\right]
\end{aligned}
$$

where $w_{1}$ and $w_{2}$ are the density-independent selective values, $\rho_{1}$ and $\rho_{2}$ are additional variables determining (with $w_{1}$ and $w_{2}$ ) the intrinsic rate of growth per generation, $K_{1}$ and $K_{2}$ are the carrying capacities and $\alpha_{1}$ and $\alpha_{2}$ are the competition coefficients.

Using equations (6) and (7), which are general, we can calculate, as before, the numerical loads for particular types of mutants.

Mutants reducing $w$ give

$$
\mathcal{N} L=\frac{\mu[1-(1-\mu)(w[1+\rho]-1)]}{[w(1+\rho)-1](1-\mu)^{2}} .
$$

Under some circumstances this load can be negative. When $w$ and $\rho$ are large it approximates to $-\mu$.

Mutants causing reduced values of $\rho$ or $K$ give

$$
\mathcal{N} L=\frac{\mu}{[w(1+\rho)-1][1-\mu]}
$$

$\alpha$-mutants give the same result when $\alpha_{1}=1, \alpha_{2}=1+s^{\prime \prime}$. However, when $\alpha_{1}=1-s^{*}, \alpha_{2}=1$ the numerical load is

$$
\mathcal{N} L \bumpeq \frac{\mu}{w(1+\rho)-1}-(1-\mu) \sqrt{\frac{\mu s^{*}}{w(1+\rho)-1}}
$$

and is negative under the conditions of the model.

\section{The timing of SELEGTION}

Both models assume that density-independent selection acts first, thereby modifying the results of the density-dependent selection. If the two forms of selection occur in the reverse order, a situation perhaps equally frequent in nature, the equations become simpler. For the first model, all the numerical loads are unchanged except that due to $w$ mutants, which becomes

$$
\frac{w \mu}{w-1}
$$

as for $K$-mutants.

Similarly in the second model, all the loads are again unchanged except that due to $w$ mutants, which becomes

$$
\frac{\mu}{(w[1+\rho]-1)(1-\mu)}
$$

as for $\rho$ and $K$ mutants. 
Thus the relative timing affects only the ability of the density-dependent factors to compensate for density-independent mortality. If densitydependent selection acts first, the $w$-loads are brought into line with the $\kappa$-loads.

\section{The biological significance of the models}

Several important points emerge from this analysis. First, there are three major classes of mutation, mutants reducing the intrinsic selective value ( $w$-mutants), mutants reducing the carrying capacity ( $K$-mutants), and mutants reducing the competitive ability ( $\alpha$-mutants). These classes are not, of course, mutually exclusive. They all produce the same conventional genetic load ( $L=\mu$ for complete recessives), as has been proved for the general case of density-dependent selection by Kimura and Ohta (1971). However, they may have markedly different effects on the population size at equilibrium. These effects demand further consideration.

Let us first consider the effects of $w$-mutants when density-independent selection acts before density-dependent selection. For $w$-mutants using the first (Michaelis) model the proportional decrease of numbers is

$$
\mathcal{N} L=\frac{\mu}{(w-1)(1-\mu)} \bumpeq \frac{\mu}{w-1}=\frac{\mu}{R_{\max }}
$$

where $R_{\max }$ is the maximum net reproductive rate (or intrinsic rate of increase per generation, which in this case is equal to $w-1)$. Values of $R_{\max }$ have been estimated for many natural and artificial populations. They vary from zero (some populations of men) to several million (some marine invertebrates; see Odum, 1971). For most higher organisms it is likely that $R_{\max }>10$. Consequently under the Michaelis model the numerical load due to $w$-mutations will be small $\left(\frac{\mu}{10}\right.$ or less $)$. If there were $10^{5}$ genes capable of producing $w$-mutants, if their effects were multiplicative, and the rate of $w$-mutation was $10^{-6}$, the proportional decrease of population size due to all of them would be $10^{-2}$ or less.

Using the second (logistic) model, the occurrence of w-mutats will usually produce an increase in population size, rather than a decrease. The numerical load is

$$
\begin{gathered}
\mathcal{N} L=\frac{\mu[1-(1-\mu)(w[1+\rho]-1)]}{(w[1+\rho]-1)(1-\mu)^{2}}=\frac{\mu\left[1-R_{\max }(1-\mu)\right]}{R_{\max }(1-\mu)^{2}} \\
\bumpeq \frac{\mu}{R_{\max }}-\mu \text { when } \mu \text { is small. }
\end{gathered}
$$

In this case $R_{\max }$ is equal to $w[1+\rho]-1$. The numerical load is thus negative when $R_{\max }(1-\mu)>1$. With $10^{5}$ loci, a mutation rate of $10^{-6}$ and $R_{\max }>10$, the total effect of mutation would be to cause approximately a 10 per cent. increase in numbers.

At first sight it may seem surprising that a disadvantageous mutant, in the process of being eliminated by selection, can produce an increase in population size. However, it is a property of the logistic equation to " overcompensate" for prior reductions in numbers. It might be suggested 
that this property disqualifies the logistic as a realistic model of population growth, were it not for the observations of Nicholson, who reported a case in which an increased larval mortality produced a correspondingly increased population of adults (Nicholson, 1950).

It is only with $w$-mutants that equilibrium numbers are affected by the timing of selection. When density-dependent selection acts first, the numerical loads due to $w$-mutants become identical to those due to $K$ - or $\rho$-mutants.

Using the Michaelis model, $K$-mutants produce a numerical load of

$$
\mathcal{N} L=\frac{\mu}{R_{\max }}+\mu .
$$

With $10^{5}$ loci, $\mu=10^{-6}$ and $R_{\max }>10$ the total numerical load will have a minimum value of $10^{-1}$. Thus the effects of $K$-mutation on population size may be appreciable.

These effects are less severe when the logistic model is used. For $K_{\text {- }}$ (or $\rho-)$ mutants,

$$
\mathcal{N} L=\frac{\mu}{R_{\max }}
$$

and the total numerical load (with $10^{5}$ loci, $\mu=10^{-6}$ and $R_{\max }>10$ ) is $10^{-2}$ or less.

Mutations causing a decrease in competitive ability ( $\alpha$-mutants) can be of two kinds. The first, in which numbers of mutants are more severely depressed by the presence of wild-type organisms than by other mutants $\left(\alpha_{1}=1, \alpha_{2}=1+s^{\prime \prime}\right)$ produces a numerical load identical to that caused by $K$-mutants. The second kind, in which the numbers of wild-type are less severely depressed by the presence of mutants than by other wild-type $\left(\alpha_{1}=1-s^{*}, \alpha_{2}=1\right)$ produces a load that is negative. For the Michaelis model

$$
\mathcal{N} L \bumpeq \frac{\mu\left[R_{\max }+1\right]-\sqrt{s^{*} \mu R_{\max }\left(R_{\max }+1\right)}}{R_{\max }-\sqrt{s^{*} \mu R_{\max }\left(R_{\max }+1\right)}}
$$

When $R_{\max }$ is large this load approximatès to $\mu-\sqrt{s^{*} \mu}$. It is negative when $s^{*}>\mu$ (i.e. under the conditions of the model).

For the logistic

$$
\mathcal{N} L \bumpeq \frac{\mu}{R_{\max }}-\frac{(1-\mu) \sqrt{\mu s^{*}}}{\sqrt{R_{\max }}}
$$

which is also negative under the conditions of the model.

Once more a disadvantageous mutant in the process of elimination can produce an increased population size. In this case the fact that $\alpha_{1}$ is less than unity indicates that the requirements (" niches ") of the two phenotypes do not exactly correspond. The presence of mutants, disadvantageous though they may be, allows the population to exploit a larger component of the environment.

Both kinds of $\alpha$-mutants have the interesting property that a population composed entirely of mutants would attain the same size as a population composed entirely of wild-type. In monomorphic populations the components of the equation containing $\alpha$ disappear.

Wallace (1970) discussing genetic load, drew a distinction between " hard selection" which necessarily reduces population numbers, and "soft 
selection " which does not. He tentatively suggested that " hard selection" is related to density-independent factors, and that " soft selection " is related to density-dependent factors. It now seems that Wallace's " soft selection" is equivalent to my $\alpha$-selection.

The conventional genetic load is dependent only upon the mutation rate. The numerical load, however, depends also on the value of $R_{\max }$, the maximum net reproductive rate. When $R_{\max }$ is small (near to zero) all types of mutants, except $\alpha_{1}$ mutants, may produce a numerical load greatly larger than the mutation rate. Mutation may then threaten the survival of the population. $R_{\max }$ measures the buffering of the population against mutation (or other disturbances), and it is an indicator of what has been called the "load space". The ratio $\frac{\mu}{R_{\max }}$ is an important component of all

the equations. Since both the parameters in this ratio are measureable, it is potentially possible to predict the effects of mutation on the equilibrium population size of any organism. Before this possibility can be realised in practice we need to know the relative frequencies of different kinds of mutants, and to find a satisfactory general model of population growth.

\section{Genetic load and the RAte of population inGrease}

The conventional definition of genetic load inevitably leads to the conclusion that $L=\mu$. This is a property of the conditions for equilibrium of gene frequency. There is, however, another relevant definition of load. Grow (1970) has remarked that it may be defined so that $W_{\max }$ in equation (1) refers to the mean fitness of a population composed only of the non-mutant genotype. He states that the two definitions usually lead to the same numerical conclusions. In the present model, however, they do not.

The reason for the discrepancy is that the value of $a$ depends upon the composition of the population. In the conventional definition we use the value obtained from a population containing mutants. In the second definition we use the value obtained from a population containing only wildtypes. The genetic load at equilibrium is then found to be zero.

Since the selective values depend upon numbers, there is no genetic equilibrium without numerical equilibrium. Thus we cannot use either definition of genetic load as a direct measure of the effect of mutation on population growth.

An appropriate measure would be the "growth load":

$$
G L=\frac{\Delta \mathcal{N}_{\max }-\Delta \mathcal{N}_{\text {mut }}}{\Delta \mathcal{N}_{\max }}
$$

where $\Delta \mathcal{N}_{\max }$ is the rate of increase of a population without mutation and $\Delta \mathcal{N}_{\text {mut }}$ is the rate of increase of a population with mutation. At joint equilibrium, of course, the "growth load" is zero.

The value of $\Delta \mathcal{N}_{\max }$ depends upon numbers, and the value of $\Delta \mathcal{N}_{\text {mut }}$ depends upon both numbers and gene-frequency. Thus when numbers and gene-frequencies are not in equilibrium there is no unique solution for $G L$. It is clear, however, that because the presence of deleterious mutants can sometimes increase the size of the population at joint equilibrium, the "growth load" can be negative. 


\section{General conclusions}

It is possible to combine the theories of ecology and population genetics, and to predict the effects of mutation on population size. Two algebraic models, making different assumptions about the control of population size, give broadly similar results. The following conclusions have emerged. They appear to be general, in the sense that they are independent of the ecological model.

1. Although all disadvantageous mutants produce the same genetic load (as conventionally defined) different types of mutants may have different effects on the population size at numerical equilibrium.

2. In relation to their effects on population size, disadvantageous mutants can be grouped into three broad classes, which are not mutually exclusive: $w$-mutants, with a reduced density-independent selective value (and consequently a reduced intrinsic rate of increase); $K$-mutants, with a reduced carrying capacity; and $\alpha$-mutants, with a reduced competitive ability.

3. The effects of $w$-mutants on population size depend upon the relative timing of density-dependent and density-independent factors.

4. In some circumstances the presence of disadvantageous mutants can cause an increase in population size, despite the fact that the mutants are eliminated by natural selection. It follows, conversely, that advantageous mutants could reduce the population size.

5. For all classes of disadvantageous mutants, the effects of mutation on population size are strongly dependent upon the value of $\frac{\mu}{R_{\max }}$ where $\mu$ is the mutation rate, and $R_{\max }$ is the maximum net reproductive rate (or intrinsic rate of increase per generation). If $R_{\max }$ is small, and approaches the value of $\mu$, all classes of mutation, except $\alpha_{1}$-mutants, may cause a severe reduction in population size, and may even threaten the survival of the population.

6. When the selective values of genotypes depend upon their frequencies (or densities) in a population, conventional genetic load measures neither the effect of mutation on the rate of increase of the population nor its effect on the total numbers at equilibrium. If the consequences of mutation are to be adequately understood, the ecological parameters must be taken into consideration.

Acknowledgments.-I am very grateful to Dr Brian Charlesworth, Dr John Endler, Mr Len Nunney, Dr Peter O'Donald and Dr Jonathan Roughgarden for critically reading the manuscript, and to the Nuffield Foundation and the Science Research Council for financial support.

\section{RefERENCES}

BARBER, H. N. 1965. The genetics of populations. Heredity, 20, 551-572.

CAIN, A. J., AND ShEPPARD, P. M. 1954. The theory of adaptive polymorphism. Am. Nat., $88,321-326$.

CHARLESWORTH, B. 1971. Selection in density-regulated populations. Ecology, 52, 469-475. CLARKE, B. 1972. Density-dependent selection. Am. Nat., 106, 1-13.

CROW, J. F. 1970. Genetic loads and the cost of natural selection. In Mathematical Topics in Population Genetics (ed. K. Kojima). Springer-Verlag, Berlin.

haldane, J. B. s. 1937. The effect of variation on fitness. Am. Nat., 71, 337-349. kimura, M., AND oHta, T. 1971. Theoretical Aspects of Population Genetics. Princeton. 
KING, C. E., AND ANDERSON, w. w. 1971. Age-specific selection. II. The interaction between $r$ and $k$ during population growth. Am. Nat., 105, 137-156.

LI, c. c. 1955. The stability of an equilibrium and the average fitness of a population. Am. Nat., 89, 281-295.

LOTKA, A. J. 1932. The growth of mixed populations: two species competing for a common food supply. 7. Wash. Acad. Sci., 22, $461-469$.

macarthur, R. H. 1962. Some generalised theorems of natural selection. Proc. natn. Acad. Sci. U.S.A., 48, 1893-1897.

MULLER, H. J. 1950. Our load of mutations. Am. 7. hum. Genet., 2, 111-176.

NICHOLson, A. J. 1950. Population oscillation caused by competition for food. Nature, Lond., $165,476-477$.

ODUm, E. P. 1971. Fundamentals of Ecology (3rd ed.). Saunders, Philadelphia.

Roughgarden, J. 1971. Density-dependent natural selection. Ecology, 52, 453-468.

tURner, J. R. G., AND williamson, M. W. 1968. Population size, natural selection and the genetic load. Nature, Lond., 218, 700.

verhulst, P. F. 1844. Recherches mathematiques sur la loi d'accroissement de la population. Mem. Acad. Roy. Bruxelles, 20, 1-52.

volterra, v. 1926. Variazioni e fluttuazioni del numero d'individui in specie animali conviventi. Mem. Accad. Lincei, ser. 6, 2, 31-113.

wallace, B. 1970. Genetic Load: Its Biological and Conceptual Aspects. Prentice-Hall, Englewood Cliffs, N.J.

WiLliamson, M. H. 1957. An elementary theory of interspecific competition. Nature, 180, $422-425$.

williamson, м. н. 1972. The Analysis of Biological Populations. Arnold, London. 